\title{
Trace Elements Interactions in Soils and Plants Due to the Wastewater Reuse
}

\section{loannis K. Kalavrouziotis*}

School of Science and Technology, Hellenic Open University, Aristotelous 18, 26 335, Patras, Greece

Treated municipal wastewater (TMWW) is not only an alternative irrigation water source. Its reuse prevents wastewater disposal into the surface waters such as rivers, lakes, seas, oceans, and thus minimizes environmental pollution. Consequently more fresh water is economized for domestic and industrial use, and crop irrigation, with the added benefit that nutrients are added to soils during the reuse. In some locations, the TMWW water is becoming more attractive because modern wastewater treatment technologies can produce water of good quality.

The TMWW is a carrier of nutrients. That can satisfy crop needs for optimal production, but in some cases may surpass real plant needs. In the long run, significant quantities of nutrients may accumulate in the soil and groundwater, but also heavy metals as well.

The elemental interactions, as all kinds of interactions, display synergism or antagonism. Synergism is the process occurring between two interacting elements, whereby the concentration increase of one element increases the level of the other. Synergism between ions is a type of interaction which could be defined as the stimulation of an ion by another one during their uptake by plants. The process of antagonism refers to the competition between essential nutrients and heavy metals occurring in the plants or in soil. Thus, the presence of a given nutrient or heavy metal in high levels may depress the concentration of another nutrient or metal. Such interactions may occur either in soil, or on root interface or within the plant.

So far existing research literature has focused on the type of interactions occurring between plants and soils after irrigated with TMWW. They have concluded that numerous interactions are continuously occurring in plants and soils with usually the synergistic ones dominating over the antagonistic. The total number of interactions, irrespective of their type i.e. synergistic or antagonistic, and independent of the plant organ, varies with the plant species. The interactions play an important role in plant nutrition by supplying primarily essential nutrients and heavy metals secondarily, the level of the supplied elements depending on the degree of elemental synergism and antagonism. The interactions contribute to soil mainly essential elements and secondarily, heavy metals, affecting favourably soil fertility. Regression analysis made possible the quantitative expression of the elemental interaction's contribution in essential elements and heavy metals to soil and plants. The quantification of the elemental contribution by the interactions showed that the interactions may supply the soil and plants with significant quantities of nutrients and heavy metals under TMWW. The occurrence of the interactions is characterized by high degree of consistency as the interactions occurring in the soil growth medium are strongly related to those occurring in the plant leaves, not only in relation to their total number, but to their type, as well. Finally, the interactions could be used for an in depth understanding of the elemental interrelationships in soils and plants, especially in relation to nutrient and heavy metal supply of the growing plant species. They could also be used for explaining the positive involvement of heavy metals in plant growth and development.

However there are several more aspects to be addressed such as the interactions between the essential elements and heavy metals, their effect on elemental accumulation in soil, and plants, the distribution of the interactions in the various plant parts (roots, leaves, shoots, edible parts), and the quantitative expression of the elemental contribution to plant and soils, under the TMWW.

These topics have not so far been within the immediate interest of the researchers.
*Corresponding author: loannis K. Kalavrouziotis, Dr. Associate Professor School of Science and Technology, Hellenic Open University, Aristotelous 18, 26 335, Patras, Greece, Tel: +302610367546; E-mail: ikalabro@eap.gr

Received March 20, 2015; Accepted March 25, 2015; Published March 30, 2015

Citation: Kalavrouziotis IK (2015) Trace Elements Interactions in Soils and Plants Due to the Wastewater Reuse. J Environ Anal Toxicol 5: e105. doi: 10.4172/2161-0525.1000e105

Copyright: (c) 2015 Kalavrouziotis IK. This is an open-access article distributed under the terms of the Creative Commons Attribution License, which permits unrestricted use, distribution, and reproduction in any medium, provided the original author and source are credited. 